\title{
Demonstration of a speckle nulling algorithm and Kalman filter estimator with a fiber injection unit for observing exoplanets with high- dispersion coronagraphy
}

Yeyuan Xin, Jorge Llop Sayson, Nikita Klimovich, Dimitri Mawet, Garreth Ruane, et al.

Yeyuan Xin, Jorge Llop Sayson, Nikita Klimovich, Dimitri Mawet, Garreth Ruane, Jacques Delorme, Nemanja Jovanovic, "Demonstration of a speckle nulling algorithm and Kalman filter estimator with a fiber injection unit for observing exoplanets with high-dispersion coronagraphy," Proc. SPIE 10703, Adaptive Optics Systems VI, $107036 Z$ (18 July 2018); doi: $10.1117 / 12.2312357$

Event: SPIE Astronomical Telescopes + Instrumentation, 2018, Austin, Texas, United States 


\title{
Demonstration of a Speckle Nulling Algorithm and Kalman Filter Estimator with a Fiber Injection Unit for Observing Exoplanets with High-dispersion Coronagraphy
}

\author{
Yeyuan Xin ${ }^{a}$, Jorge Llop Sayson ${ }^{a}$, Nikita Klimovich $^{a}$, Dimitri Mawet ${ }^{a}$, Garreth Ruane $^{a}$, \\ Jacques Delorme ${ }^{a}$, and Nemanja Jovanovic ${ }^{a}$ \\ ${ }^{a}$ California Institute of Technology, Exoplanet Technology Lab, Department of Astronomy, \\ 1200 E. California Blvd., Pasadena, USA, 91125
}

\begin{abstract}
High-dispersion coronagraphy (HDC) combines high contrast imaging techniques with high spectral resolution spectroscopy to observe exoplanets and determine characteristics such as chemical composition, temperature, and rotational velocities. It has been demonstrated in lab that with monochromatic light, a fiber injection unit (FIU), in which an optical fiber is used to couple to light from the exoplanet, could be used to direct exoplanet light to a high-resolution spectrograph, with robust performance and speckle suppression that exceeds conventional image-based speckle nulling. We now demonstrate in lab a FIU based speckle nulling scheme with a Kalman filter estimator. We currently find that speckle nulling with a Kalman filter is more stable and outperforms traditional speckle nulling by $10 \%$ in suppression in the presence of white detector noise.
\end{abstract}

Keywords: vortex coronagraph, fiber injection unit, speckle nulling, Kalman filter, spectrograph, exoplanet characterization

\section{INTRODUCTION}

Direct imaging of exoplanets is one method of finding and characterizing exoplanets through spectroscopy, which can help identify characteristics such as chemical composition, temperature, and rotation. However, this method faces many technical challenges in order to achieve the level of contrast required to observe exoplanets bound to their host stars. Firstly, telescope performances are limited by the effect of turbulence in the atmosphere on the incoming light. Adaptive optics (AO) systems use wavefront sensors and deformable mirrors to correct for the atmospheric distortions. There remain, however, imperfections and residual bright spots of starlight, called speckles, in the image. These speckles can have similar characteristics, such as size and brightness, to exoplanets, so the goal is to minimize them as much as possible to maximize the planet signal.

Another problem is that, without manipulation, the massive amount of light from the star obscures the light from the exoplanet. To address this issue, coronagraphs, devices that suppress light from the star in the image, are used to allow planets that are much dimmer than the star to be imaged. However, as coronagraphs are very sensitive to the residual aberrations not corrected by the AO system, it is still very difficult to achieve such contrast with a coronagraph, and additional solutions such as the fiber injection unit (FIU) are needed to detect and characterize planets. ${ }^{1}$

The High Contrast Spectroscopy Testbed (HCST) at the Caltech Exoplanet Technology Lab is a facility to test high contrast imaging and spectroscopy technologies for future applications. ${ }^{2}$ The team demonstrated last summer that, in monochromatic (one wavelength) light, an FIU, in which an optical fiber is used to couple to light from the exoplanet, could be used to direct exoplanet light to a high-resolution spectrograph. ${ }^{1}$ Since the injection efficiency of the FIU is given by the overlap integral

$$
\eta=\frac{\left|\iint H E_{11}(x, y) A^{*}(x, y) d x d y\right|^{2}}{\iint\left|H E_{11}(x, y)\right|^{2} d x d y \iint|A(x, y)|^{2} d x d y}
$$

Further author information: (Send correspondence to Yeyuan Xin)

Yeyuan Xin.: E-mail: yxin1025@gmail.com

Adaptive Optics Systems VI, edited by Laird M. Close, Laura Schreiber,

Dirk Schmidt, Proc. of SPIE Vol. 10703, 107036Z • @ 2018 SPIE

CCC code: $0277-786 X / 18 / \$ 18 \cdot$ doi: $10.1117 / 12.2312357$

Proc. of SPIE Vol. 10703 107036Z-1 

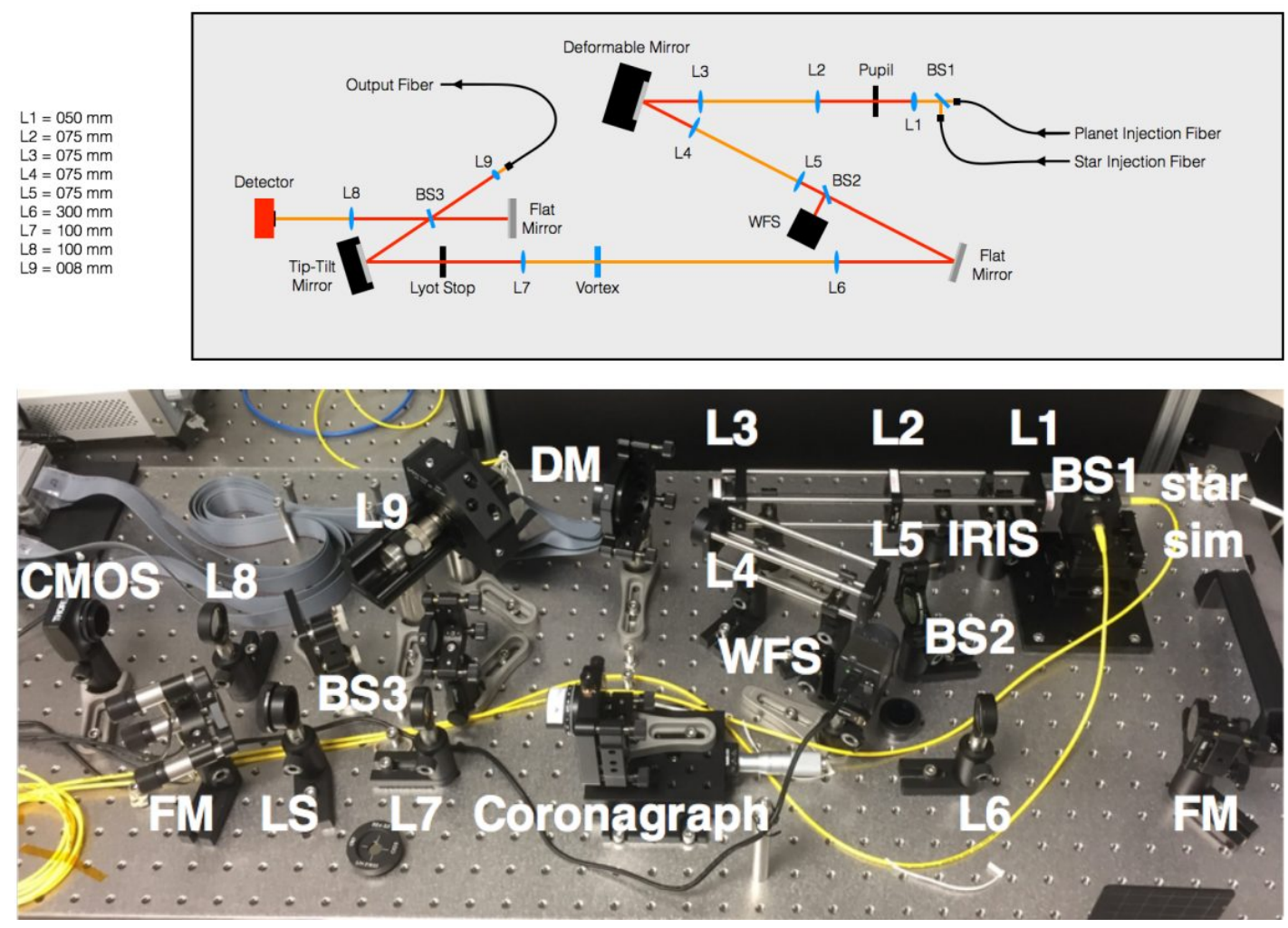

Figure 1. Diagram of the experimental setup. Light is input through the star injection fiber. The $12 \mathrm{x} 12$ actuator DM (Boston Micromachines Multi-DM) is used for wavefront control. The vortex coronagraph suppresses the central PSF. The tip tilt mirror is used to inject a speckle (to be nulled) into the FIU, and the power coupled into the FIU is measured by a photodetector. An additional scientific camera is used to monitor the image. ${ }^{3}$

the FIU provides additional degrees of freedom to null residual starlight that couples into the fiber, as nonzero asymmetric fields will not couple into the fiber, providing additional gains in starlight suppression schemes. ${ }^{1}$

We present such a starlight suppression scheme, called speckle nulling, aided by a Kalman filter estimator, and laboratory results from HCST.

\section{LABORATORY SETUP}

The transmissive testbed of HCST consists of a telescope simulator, followed by an AO system, a coronagraph, and the FIU prototype (see Figure 1). The telescope simulator images simulated star and off-axis planetary sources, generated by a Thorlabs $635 \mathrm{~nm}$ laser diode and a filtered NKT Photonics supercontinuum white light source (narrowband filter centered at $650 \mathrm{~nm}$ ), respectively. The AO system is made up of a Boston Micromachines 144-actuator MEMS DM, pellicle BS, and a Shack-Hartmann wavefront sensor (Thorlabs AOK1UM01).

The AO system is followed by a relay of a focusing lens, focal-plane mask, collimating lens, and Lyot stop. The focal plane mask is a vortex coronagraph, a phase-based coronagraph that rejects light by through a phase singularity at the center of the device that diffracts light outwards to be blocked by a Lyot stop. This provides an effective inner-working angle of $1.7 \lambda / D$ at which $50 \%$ off-axis transmission is obtained.

Downstream from the coronagraph's Lyot stop lies the FIU. We use a three-axis TTM from Newport, actuated by computer-controlled Thorlabs piezoactuators. The FIU BS is a 50\%/50\% BS.

The tracking camera is a CMOS sensor from Thorlabs. The SMF is mounted on a Newport Post-mount Singlemode Fiber Aligner. The CC and other optical elements are off-the-shelf Newport and Thorlabs products. 
For the purposes of this demonstration, we used a Femto OE-200-SI Fast Optical Power Meter in lieu of the high-resolution spectrograph. For science observations, the beam would be split such that the spectrograph can take science data at the same time that a power meter can be used for sensing for starlight suppression.

\section{SPECKLE NULLING SCHEME}

Speckle nulling works by destructively interfering an intentionally generated anti-speckle with an existing speckle.

A sinusoid with amplitude $h_{0} \ll \lambda$ in the pupil plane will translate into a pair of conjugated anti-speckles in the image plane due to the Fourier transform relationship between the pupil and image plane. ${ }^{4}$ In each iteration, we apply a cosine pattern to the DM surface with height

$$
h=\frac{h_{0}}{4}[\cos (2 \pi \vec{\xi} \cdot \vec{r}+\alpha)]
$$

where $h_{0} / 2$ is the maximum surface height, $\vec{\xi}$ is the spatial frequency vector and the speckle location in the image plane, $\vec{r}$ is the position vector in the pupil plane, and $\alpha$ is a constant phase offset. The intensity, position, and phase of the speckle are controlled by $h_{0}, \vec{\xi}$, and $\alpha$, respectively.

\subsection{Calibration}

The correspondence between the parameters of the sinusoid as applied to the DM and their effects on the speckle must be calibrated before running speckle nulling. The correspondence in the image plane of changing $\vec{\xi}$ as applied to the DM is calibrated by applying a sequence of speckles with different values for the magnitude and the direction of $\vec{\xi}$, and using the camera to measure where the speckles are generated on the image plane.

The correspondence of $h_{0}$ on the intensity of the speckle coupled through the fiber can similarly be calibrated by applying a speckle at the fiber location with varying values of $h_{0}$ (using four different phases to account for the effects of phase) and measuring its effects on the light coupled through the fiber.

The results of the calibrations are saved in lookup tables for use during speckle nulling.

\subsection{Nulling}

In order to null a speckle that is on the fiber, the location is first taken from the camera, and the appropriate $\vec{\xi}$ is determined from the calibration tables.

Then, a "probe" speckle is applied at the fiber location (with a DM amplitude that is smaller than the corresponding amplitude of the speckle), and a power reading is made at four different phases. The readings are Fourier transformed using FFT, and converted through the calibration tables in order to determine the amplitude $\left(h_{0}\right)$ and phase $(\alpha)$ of the anti-speckle needed to null the speckle.

After the required anti-speckle is applied to the DM, the procedure of probing the fiber and applying the required anti-speckle is repeated continuously as desired. An example of the image before and after speckle nulling and the corresponding applied DM shape is shown in Figure 2.

\section{KALMAN FILTER ESTIMATOR}

A Kalman filter estimator, which synthesizes measurements and control history to obtain the best estimation of the speckle amplitude and phase, can be used to improve performance from simply using measurements alone. We present the construction and execution of the Kalman filter for speckle nulling through the FIU. 

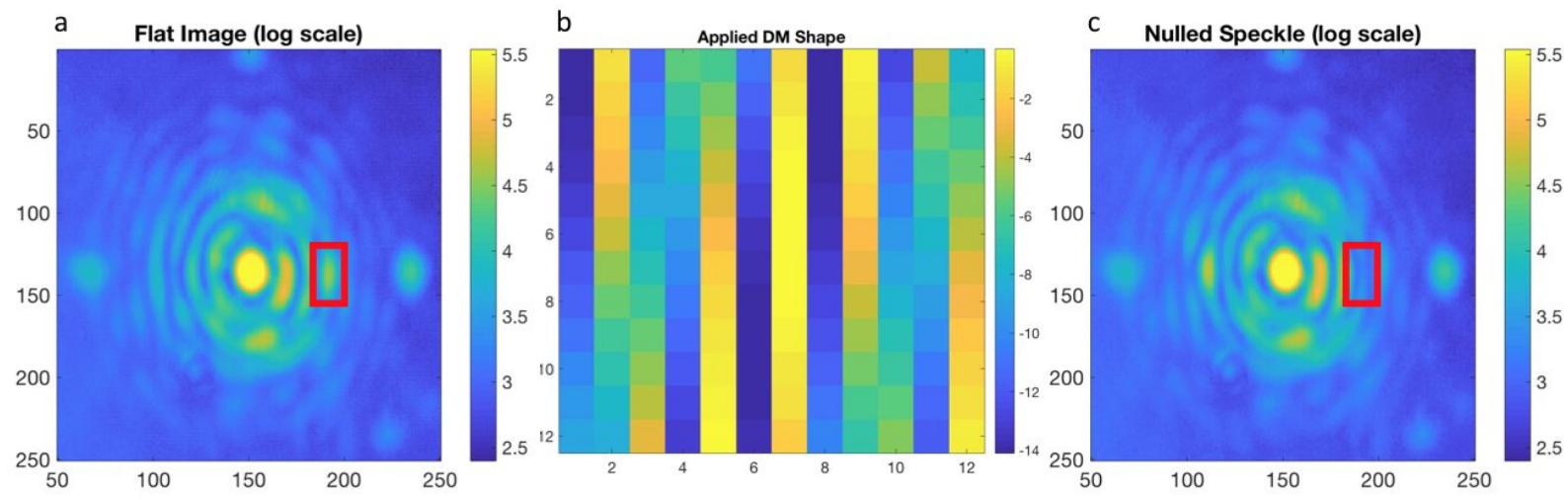

Figure 2. Example camera images showing a.) the speckle before running the algorithm, b.) The DM shape applied to create the anti-speckle that nulls the initial speckle, c.) the nulled speckle afterwards.

Table 1. Kalman Filter State Matrices

\begin{tabular}{|c|c|l|}
\hline & Definition & Function \\
\hline \hline 1 & $\Phi=I$ & state transition matrix \\
\hline 2 & $\Gamma=I$ & propagation matrix \\
\hline 3 & $H_{k}=I$ & linear observation matrix \\
\hline 4 & $z_{k}=\left(\begin{array}{c}\psi \\
A\end{array}\right)$ & measurement \\
\hline 5 & $x=\left(\begin{array}{c}\hat{\psi} \\
\hat{A}\end{array}\right)$ & estimation \\
\hline 6 & $u=\left(\begin{array}{c}\phi \\
h_{0}\end{array}\right)$ & control input \\
\hline 7 & $P_{0}=\left(\begin{array}{cc|}\sigma_{\phi i}^{2} & 0 \\
0 & \sigma_{A_{i}^{2}}^{2}\end{array}\right)$ & initial covariance matrix \\
\hline 8 & $Q=\left(\begin{array}{cc}\sigma_{\phi p}^{2} & 0 \\
0 & \sigma_{A_{p}^{2}}^{2}\end{array}\right)$ & process noise matrix \\
\hline 9 & $R=\left(\begin{array}{cc}\sigma_{\phi m}^{2} & 0 \\
0 & \sigma_{A_{m}^{2}}^{2}\end{array}\right)$ & measurement noise matrix \\
\hline
\end{tabular}

\subsection{State Matrices}

We define the following matrices that describe the state and propagation of our system in Table 1 . The functions of each of the matrices are as follows:

1. Captures known changes in the state between iterations. In our system, we assume that the state remains constant.

2. Relates control input to its expected effect on the state.

3. Relates measurements to what they indicate about the state.

4. Measurement of the phase and amplitude of the speckle.

5. Estimation of the phase and amplitude of the speckle.

6. Control input. To null the speckle, the following control input is applied.

$$
u_{k}=\left(\begin{array}{c}
\phi \\
h_{0}
\end{array}\right)=-\left(\begin{array}{c}
\hat{\psi} \\
\hat{A}
\end{array}\right)=-\hat{x}_{k}(+)
$$


7. Initial covariance matrix (uncertainties on initial state guess).

8. Expected noise from processes. Following Groff et al, we assume that process noise is dominated by DM control error. ${ }^{5}$

9. Expected noise from measurement (sensors), such as dark current, read noise, and photon noise. ${ }^{5}$

\subsection{Process}

For each iteration, the following steps are completed.

1. Make a best guess at state before measurement, based on information from state transition matrix (known changes) and on propagated control input.

2. Calculate error covariance of best guess at state before measurement.

3. Calculate the optimal gain.

4. Take a measurement.

5. Update the best guess given the measurement information.

6. Calculate the error covariance of the updated best guess.

7. Apply control input based on the updated best guess.

8. Repeat from step 1.

The standard Kalman filter equations corresponding to the steps described above are given in Table 2, for the state, $\hat{x}_{k}$, the covariance, $P_{k}$, and the gain, $K_{k}$, are as follows, for each $k^{t h}$ iteration. $^{5}$

Table 2. Kalman Filter Equations

\begin{tabular}{|c|c|l|}
\hline Step & Equation & Function \\
\hline 1 & $\hat{x}_{k}(-)=\Phi_{k-1} \hat{x}_{k-1}(+)+\Gamma_{k-1} u_{k-1}$ & state estimate extrapolation \\
\hline 2 & $P_{k}(-)=\Phi_{k-1} P_{k-1}(+) \Phi_{k-1}^{T}+Q_{k-1}$ & covariance estimate extrapolation \\
\hline 3 & $K_{k}=P_{k}(-) H_{k}^{T}\left[H_{k} P_{k}(-) H_{k}^{T}+R_{k}\right]^{-1}$ & filter gain computation \\
\hline 5 & $\hat{x}_{k}(+)=\hat{x}_{k}(-)+K_{k}\left[z_{k}-H_{k} \hat{x}_{k}(-)\right]$ & state estimate update \\
\hline 6 & $P_{k}(+)=\left[P_{k}(-)^{-1}+H_{k}^{T} R_{k}^{-1} H_{k}\right]^{-1}$ & covariance estimate update \\
\hline
\end{tabular}

\section{RESULTS}

The results of a typical speckle nulling run without and with the Kalman filter are compared below.

The raw contrast readings (including readings from the "probe" speckles) are shown in Figure 3a, with only the first 100 (out of 500) readings shown for visibility. The achieved raw contrast, as determined by averaging over the final 400 readings, was $10 \%$ better in the case of the filtered run. With this method of speckle nulling, science data may be taken concurrently and thus continuously. The corresponding readings at only the applied solutions are shown in Figure 3b. In this case, the filtered run resulted in an $8.4 \%$ improvement.

Additionally, we examine the amplitude measurements of both runs (as determined using the method described in Section 3.2), as well as the amplitude estimations from the Kalman filter, as shown in Figure 4. We see that the estimator is more stable, as it helps reject detector noise, and ultimately achieves a better null. 


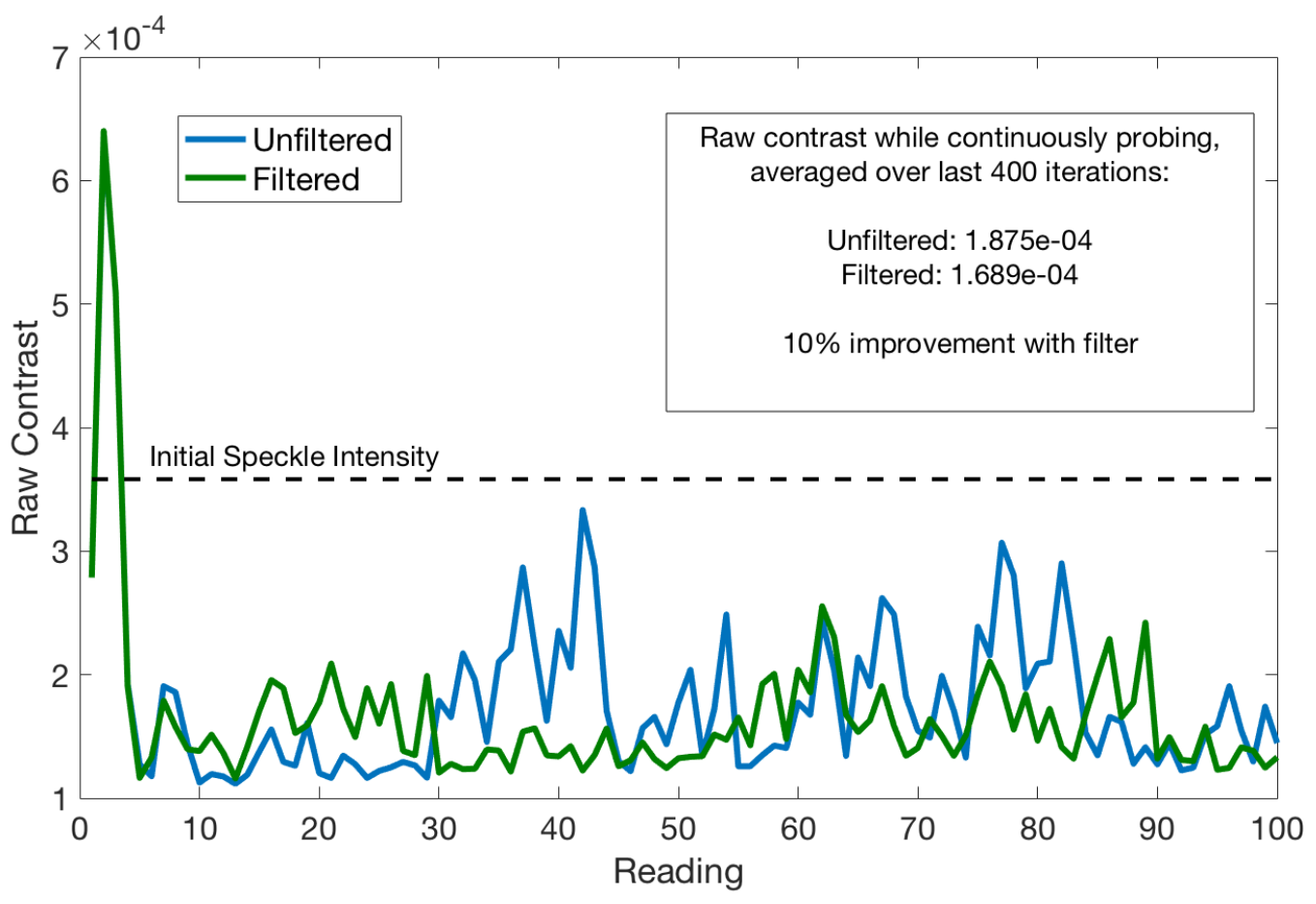

a.)

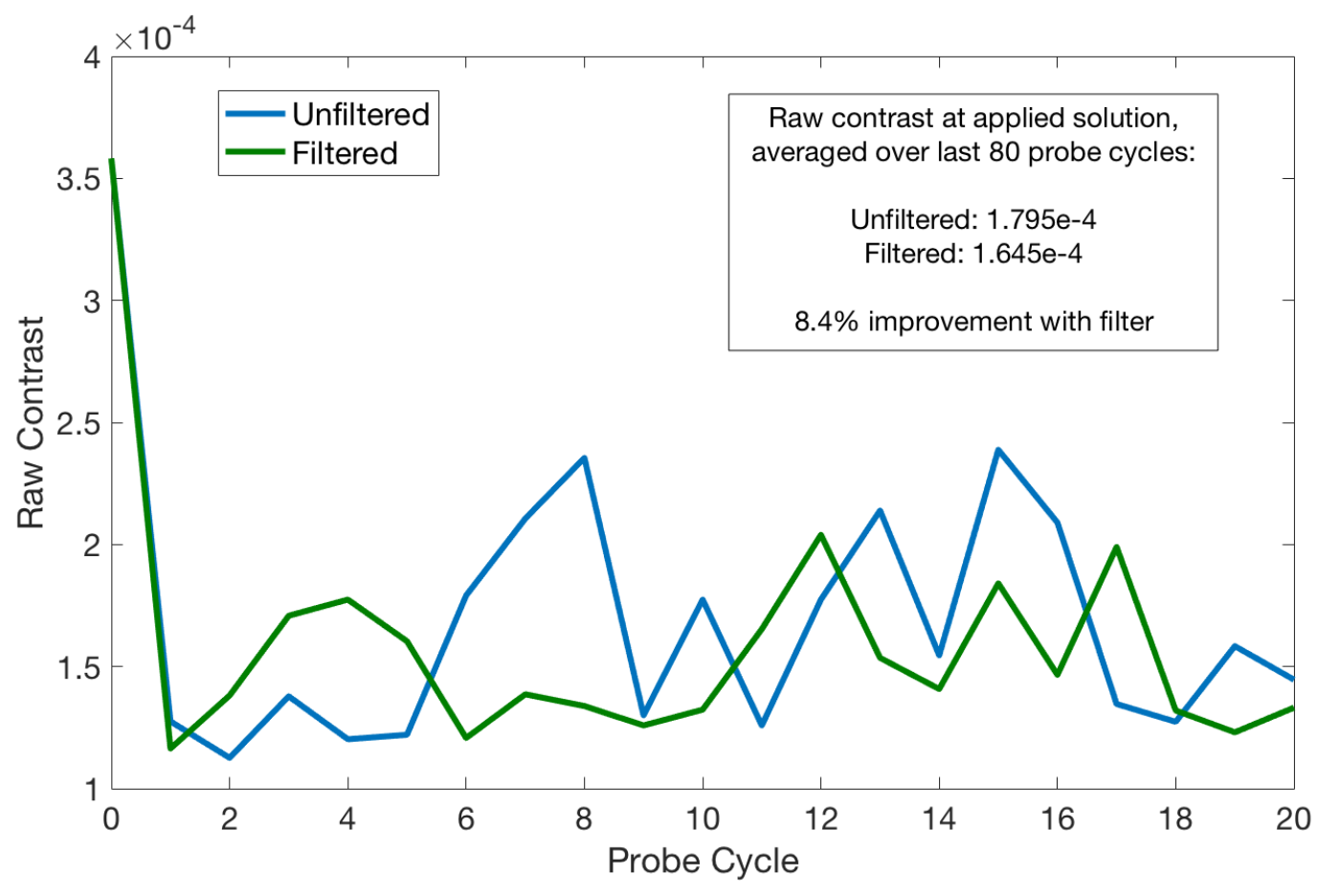

Figure 3. a.) Raw contrast readings (including while the phase is probed with the DM) through the fiber for speckle nulling with and without the Kalman filter. Only the first 100 out of the 500 total iterations are show for visibility, but the trend continues. The Kalman filter achieved a $10 \%$ better null than using measurements alone. b.) Corresponding raw contrast readings only after applying the solution (every 5 th reading of the data shown above). In this case, the Kalman filter achieved a $8.4 \%$ better null than using measurements alone. 


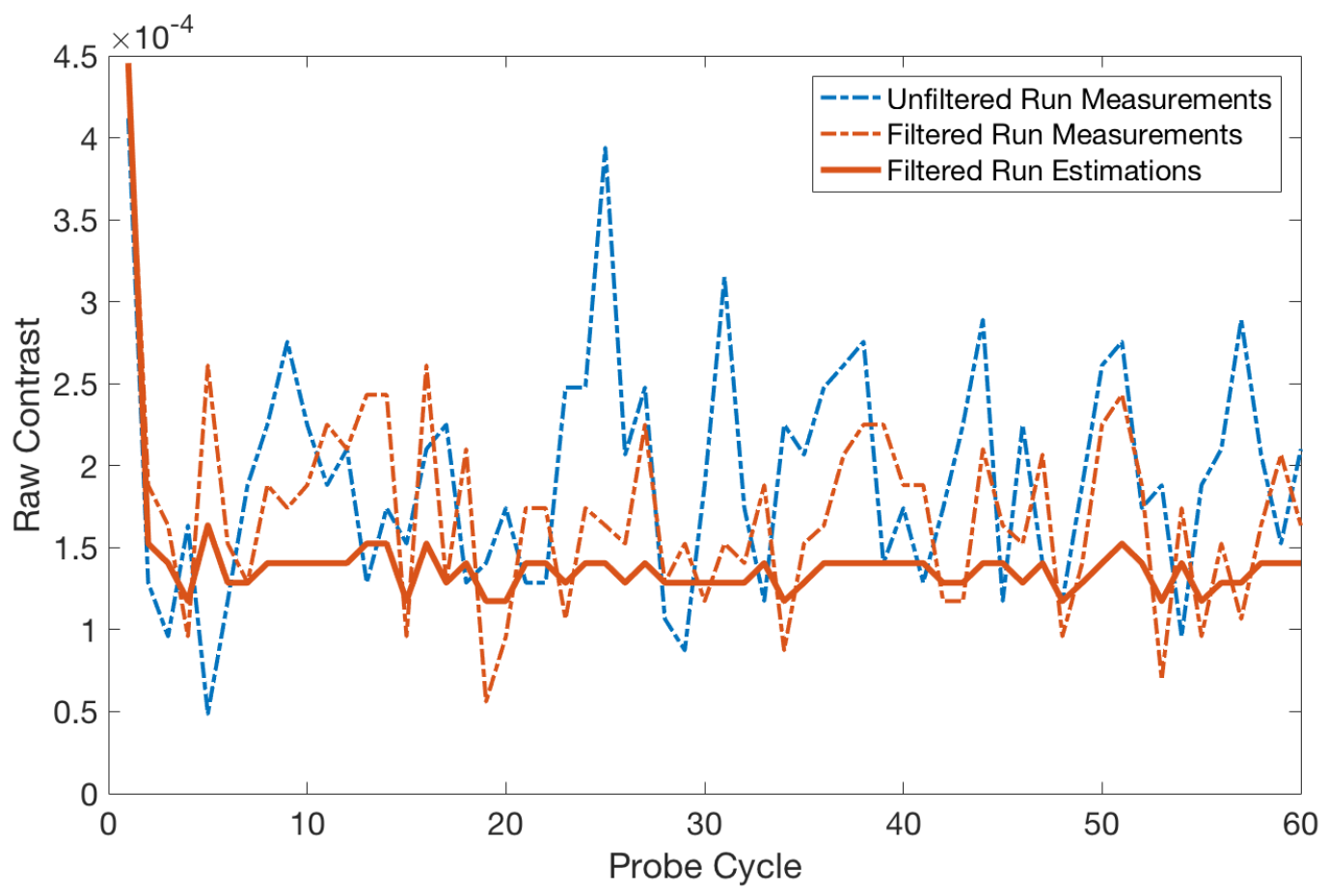

Figure 4. Raw contrast measurements, as calculated using four probes of different phases. The Kalman filter estimator helps reject detector noise and ultimately achieves a better null.

\section{CONCLUSIONS AND FUTURE WORK}

We present a speckle nulling scheme that can be used to suppress stray starlight that couples into a fiber injection unit, and make use of a Kalman filter estimator, which synthesizes measurements and control history to provide the best estimates of the speckle nulling parameters. We show in lab that the filter currently provides a $10 \%$ improvement in the regime where instrumental noise limits the nulling.

We note that the speckle was faint and close to the nulling floor to begin with, and that the Kalman filter may result in a more significant improvement if starting with a brighter, artificial speckle. We also predict that the Kalman filter will help stabilize against dynamic variations such as those introduced by air turbulence. Additionally, this Kalman filter estimation process provides a framework for predictive atmospheric control, as the residuals can be used to fit a simple, one-mode model of the atmosphere's effects at the fiber location. Future work is needed to address and test these predictions.

These tests were conducted on the transmissive testbed of HCST. Future work on speckle nulling on a singlemode fiber with Kalman filtering may be conducted on the newly operational reflective testbed of the HCST, ${ }^{2}$ which has higher stability and lower wavefront error, in order to better simulate telescope conditions and test starlight suppression techniques for high contrast spectroscopy of exoplanets.

\section{ACKNOWLEDGMENTS}

The authors would like to acknowledge the financial support of the Caltech Student Faculty Program (SFP) office and the Jean Dixon SURF Fellowship.

\section{REFERENCES}

1. D. Mawet, G. Ruane, W. Xuan, D. Echeverri, N. Klimovich, M. Randolph, J. Fucik, J. K. Wallace, J. Wang, G. Vasisht, R. Dekany, B. Mennesson, E. Choquet, J.-R. Delorme, and E. Serabyn, "Observing Exoplanets 
with High-dispersion Coronagraphy. II. Demonstration of an Active Single-mode Fiber Injection Unit," 838, p. 92, Apr. 2017.

2. J. R. Delorme, D. Mawet, J. Fucik, J. K. Wallace, G. Ruane, N. Jovanovic, N. S. Klimovich, J. D. Llop Sayson, R. Zhang, Y. Xin, R. Riddle, R. Dekany, J. Wang, E. Choquet, W. Xuan, D. Echeverri, M. Randolph, G. Vasisht, and B. Mennesson, "High-contrast spectroscopy testbed for segmented telescopes," Proc.SPIE 10400, pp. $10400-10400-7,2018$.

3. "Ay105 lab experiment \#6 fundamentals of adaptive optics." http://www.astro.caltech.edu/ dmawet/ teaching/2016-2017/ay105-lab-experiment_lab6_2.pdf, 2017. Accessed: 2018-07-01.

4. F. Malbet, J. W. Yu, and M. Shao, "High-Dynamic-Range Imaging Using a Deformable Mirror for Space Coronography," 107, p. 386, Apr. 1995.

5. T. D. Groff and N. Jeremy Kasdin, "Kalman filtering techniques for focal plane electric field estimation," Journal of the Optical Society of America A 30, p. 128, Jan. 2013. 